\title{
Immunohistological expression of cytochrome P450 1A2 (CYP1A2) in the ovarian follicles of prepubertal and pubertal rat
}

\author{
Jong-Chan Hwang ${ }^{1}$, Byung-Joon Park ${ }^{1}$, Hwan-Deuk Kim ${ }^{1,2}$, Su-Min Baek ${ }^{1}$, Seoung-Woo Lee ${ }^{1}$, \\ Ryoung-Hoon Jeon ${ }^{3}$, Min Jang ${ }^{1}$, Seul-Gi Bae ${ }^{1}$, Sung-Ho Yun ${ }^{1}$, Jin-Kyu Park ${ }^{1}$, Young-Sam Kwon ${ }^{1}$, \\ Seung-Joon $\mathrm{Kim}^{1}$ and Won-Jae Lee ${ }^{1, *}$ \\ ${ }^{1}$ College of Veterinary Medicine, Kyungpook National University, Daegu 41566, Korea \\ ${ }^{2}$ Department of Veterinary Research, Daegu Metropolitan City Institute of Health \& Environment, Daegu 42183, Korea \\ ${ }^{3}$ College of Veterinary Medicine, Gyeongsang National University, Jinju 52828, Korea
}

Received December 3, 2020

Revised December 11, 2020

Accepted December 14, 2020

\section{*Correspondence}

Won-Jae Lee

E-mail: iamcyshd@knu.ac.kr

ORCID

https://orcid.org/0000-0003-1462-7798

\begin{abstract}
Cytochrome P450 1A2 (CYP1A2) is a member of the cytochrome P450 superfamily enzymes in mammals and plays a major role in metabolizing endogenous hormones in the liver. In recent days, CYP1A2 expression has been found in not only the liver but also other tissues including the pancreas and lung. However, little information is available regarding the expression of CYP1A2 in the ovary, in spite of the facts that the ovarian follicle growth and atresia are tightly associated with controls of endocrine hormonal networks. Therefore, the expression of CYP1A2 in the ovaries of prepubertal and pubertal rats was investigated to assess its expression pattern and puberty-related alteration. It was demonstrated that the expression level of CYP1A2 was significantly $(p<0.01)$ higher in the pubertal ovaries than prepubertal counterparts. At the ovarian follicle level in both groups, whereas CYP1A2 expression was less detectable in the primordial, primary and secondary follicles, the strongly positive expression of CYP1A2 was localized in the granulosa cell layers in the antral and pre-ovulatory follicles. However, the ratio of CYP1A2-positive ovarian follicle was significantly $(p<0.01)$ higher in the ovary of pubertal group $(73.1 \pm 3.1 \%)$ than prepubertal one $(41.0 \pm 10.5 \%)$. During the Immunofluorescence, expression of CYP1A2 was mainly localized in Fas-positive follicles, indicating the atretic follicles. In conclusion, these results suggested that CYP1A2 expression was mainly localized at the atretic follicular cells and affected by the onset of puberty. Further study is still necessary but we hypothesize that CYP1A2 expresses in the atretic follicles to metabolize residue of the reproductive hormones. These findings may have important implications for the fields of reproductive biology of animals.
\end{abstract}

Keywords: cytochrome P450 1A2, follicle atresia, ovary, puberty

\section{INTRODUCTION}

Cytochrome P450 is the most important enzyme in the body and involved in the biotransformation of endogenous substances and exogenous chemicals (Dey et al., 1999). In particular, cytochrome P450 1A2 (CYP1A2) is 
a member of the cytochrome P450 superfamily enzymes in mammals as the mono-oxygenase and plays a major role in biotransformation of a wide range of exogenous chemicals, pollutants and drugs via hydroxylation, demethylation, epoxidation, oxidation and quinol formation of compounds (Dey et al., 1999; Sugiyama et al., 2019; Lu et al., 2020). Furthermore, it primarily metabolizes endogenous hormones with respect to progesterone to $6 \beta$-hydroxyl progesterone by hydroxylation, estrone to 2- or 4-hydroxyestrone by hydrolysis and estradiol to estriol by hydroxylation in humans (Mikhailova et al., 2006; Sugiyama et al., 2019; Lu et al., 2020). CYP1A subfamily contains only two functional isoforms as CYP1A1 and CY$\mathrm{P} 1 \mathrm{~A} 2$, and it has been addressed that CYP1A2 is expressed mainly in the liver; whereas the expression of CYP1A1 is mainly distributed outside the liver and involves the metabolism for aromatic hydrocarbon, CYP1A2 is expressed in the liver and metabolizes the aromatic amines and heterocyclic compounds (Wijnen et al., 2007; Lee et al., 2012; Lu et al., 2020). However, in recent days, the expression of CYP1A2 is identified in the pancreas and lung as well (Vukovic et al., 2016). Likewise, the detection of CYP1A2 expression in other tissues has been investigating to understand its function at each organ.

Puberty is regarded as the developmental stage when reproductive capacity is accomplished and sexual maturation completed under controls of complex endocrine networks, known as the hypothalamic-pituitary-gonadal (HPG) axis; it has been known that several hormones such as kisspeptin, gonadotropin-releasing hormone (GnRH), folliclestimulating hormone (FSH), luteinizing hormone (LH) and estradiol are tightly associated to the puberty-related physical changes (Mayer et al., 2010; Uenoyama et al., 2018; Kim, 2019). In rodents, external signs of puberty with respect to vaginal opening (VO) and cornification of vaginal epithelial cells are highly associated with the mature ovary and first ovulation due to the rise in estradiol levels. Therefore, $\mathrm{VO}$ in the rodent is regarded as a reliable marker for the onset of puberty and its observation is widely used because of non- or minimally-invasive procedures to assess the onset of puberty (Gaytan et al., 2017). Of particular, it is reported that VO in Spraque Dawley (SD) rat occurs on postnatal day (PND) 37-39, indirectly indicating the onset of puberty and the first ovulation (Rivest, 1991).

The follicular atresia is defined as the breakdown of the ovarian follicles including the oocyte, granulosa cells and internal/external theca cells, and its apoptotic process is hormonally controlled throughout a female mammals' life; the limited numbers of developing follicles, less than $1 \%$, are allowed for ovulation but the rest, remaining $99 \%$, undergo atresia at the various developing stages of ovarian follicle (Yu et al., 2004; Zhou et al., 2019). Among component of the ovarian follicle, survival or death of the granulosa cells plays an important role in normal follicle development with serving essential molecules for follicular growth/maintenance or follicle atresia related with hormonal imbalance between estrogen and progesterone in the follicular fluid, respectively (Yu et al., 2004; Li et al., 2016). In particular, deprivation of growth factors or activation of cell death molecules in regards to death ligands/receptors, pro-apoptotic protein and cytokines are regarded as the main cause of apoptosis of the granulosa cells; the death molecules include Fas, Fas ligand (FasL), tumor necrosis factor (TNF)- $\alpha$, TNF receptor (TNFR), TNF $-\alpha$ related apoptosis inducing ligand (TRAIL), TRAIL receptor (TRAILR) and Bcl-2 protein family (Matsuda et al., 2012; Zhou et al., 2019).

Although CYP1A2 is mainly expressed as a hepatic enzyme for metabolism of endogenous hormones, it can potentially present in other tissues. Specially, little information is available regarding the expression of CYP1A2 in the female reproductive system, in spite of the fact that the ovarian cycle is tightly and directly related with reproductive hormones. Moreover, the alteration of ovarian CYP1A2 expression depending on the onset of puberty is not understood yet. Therefore, the primary objective of the present study is to clarify the expression of CYP1A2 in the prepubertal and pubertal ovaries of female SD rats with observing different ovarian follicle stages.

\section{MATERIALS AND METHODS}

\section{Ethics statement}

All procedures for animal experiments were approved by the Institutional Animal Care Use Committee at Kyungpook National University (approval number: KNU20190159).

\section{Chemicals and media}

All chemicals and media were purchased from Thermo Fisher Scientific (Waltham, MA, USA), unless otherwise specified. 


\section{Animal housing and experiment groups}

The 21-day-old (PND 21) female SD rats (mean body weight: $58.1 \mathrm{~g}$; total $\mathrm{n}=16$ ) were housed in the plastic cages ( $40 \times 32 \times 17 \mathrm{~cm} ; 3-4$ animals/cage), maintained for temperature at $23 \pm 2^{\circ} \mathrm{C}$, humidity at $50-80 \%$ and approximately $12 \mathrm{~h}$ light/dark cycle. The standard feed (Jeil Feed Co., Ltd., Daejeon, Korea) and tap water were provided ad libitum. The SD rats were acclimatized for 3 days (PND 24). Then the rats were sacrificed at PND 24 or Day 51 as prepubertal group $(n=8)$ or pubertal group $(n=8)$, respectively.

\section{Sacrifice of animals and sampling}

The animals were anesthetized with isoflurane inhalation in the closed chamber and exsanguinated via the right atrium. The ovary and liver tissues of each group were collected, trimmed, snap-frozen into liquid nitrogen $\left(\mathrm{LN}_{2}\right)$ and stored in deep freezer $\left(-80^{\circ} \mathrm{C}\right)$ for western blotting (each $\mathrm{n}=5$ ) or fixed with $4 \%$ paraformaldehyde (Duksan chemical, Korea) for hematoxylin \& eosin staining (H\&E) and immunohistochemistry (IHC) (each $n=3)$.

\section{Body weight and vaginal opening assessment}

Before sacrificing the animals, body weight (BW) and vaginal opening (VO) of all animals was assessed by using a weighing machine or recognizing the vaginal hole over the urethra in accordance with the previous article, respectively (Rivest, 1991).

\section{Western blotting for quantitative expression of CYP1A2}

The western blotting was conducted in accordance with the previous article to assess CYP1A2 expression in the ovary and liver (positive control) (Lee et al., 2019). The snap-frozen ovaries and livers were homogenized, lysed with a radioimmunoprecipitation assay (RIPA) buffer supplemented a proteinase inhibitor and centrifugated at $14,000 \mathrm{rpm}$ for $5 \mathrm{~min}$ at $4^{\circ} \mathrm{C}$. The supernatants were carefully collected and quantified for the total amount of protein using a Bicinchoninic Acid Protein Assay Reagent Kit. The sodium dodecyl sulfate-polyacrylamide gel electrophoresis (SDS-PAGE) was conducted using $10 \mu \mathrm{g}$ of total protein for protein separation. The gels were transferred onto polyvinylidene difluoride (PVDF) membranes (Millipore, MA USA), followed by blocking the membranes with $3 \%$ bovine serum albumin (BSA) for $1 \mathrm{~h}$ at room temperature (RT). Thereafter, membranes were incubated with a mouse polyclonal anti-CYP1A2 antibody (1:500 dilution with 1\% BSA; Santa Cruz Biotechnology, TX, USA) for overnight at $4{ }^{\circ} \mathrm{C}$. As a reference, a mouse polyclonal anti-Glyceraldehyde 3-phosphate dehydrogenase (antiGAPDH; 1:1,000 dilution with $1 \%$ BSA) was involved. The membranes were then washed with tris buffer saline containing $0.1 \%$ tween-20 (TBST), incubated with a horseradish peroxidase conjugated goat anti-mouse IgG (1:3,000 dilution with TBST) for $1 \mathrm{~h}$ at RT and developed on X-ray films (AGFA, Belgium) using an enhanced chemiluminescence (ECL) kit. Image J software (National Institutes of Health, USA) was employed for quantitative analysis of the intensities of developed bands. The expression level of CYP1A2 was relatively normalized against that of GAPDH.

\section{Hematoxylin \& eosin staining and immunohistochemistry}

The preparation for histological observation was followed with the previous article (Kim, 2019). In brief, fixed ovaries from each group were dehydrated, embedded in paraffin and sectioned into $5 \mathrm{~mm}$ thick using a microtome (Leica Microsystems, Germany). The H\&E staining was then conducted in a routine manner to observe the presence of the corpus lutea. In case of IHC, the slide sections were deparaffinized, treated with $0.01 \mathrm{M}$ citrate buffer ( $\mathrm{pH} 6.0$ ) at $95^{\circ} \mathrm{C}$ for $30 \mathrm{~min}$ for antigen retrieval, cooled at RT for 60 min, treated with $3 \%$ hydrogen peroxide for $30 \mathrm{~min}$, washed with phosphate buffered saline with triton-X (PBST) and blocked with $2 \%$ normal horse serum (Histostain-plus kit) for $1 \mathrm{~h}$ at RT. Thereafter, the slides were incubated with a mouse polyclonal antiCYP1A2 antibody (1:50 dilution with 2\% normal horse serum) at $4^{\circ} \mathrm{C}$ for overnight, incubated with a biotinylated secondary antibody (Histostain-plus kit; 1:200 dilution with PBS) at RT for 90 min, treated with an $\mathrm{ABC}$ solution (Histostain-plus kit) at RT for $60 \mathrm{~min}$, reacted with a 3,3' -diaminobenzidine (DAB) kit (Vector Laboratories, CA, USA) and counterstained with hematoxylin; all slides were reacted with $D A B$ for equal time. When the slides were observed using Moticam Pro 20A (Motic, Hong Kong), brownish color on the tissue was determined as positive expression of CYP1A2.

\section{Classification of ovarian follicles}

The ovarian follicles in IHC were classified, in accordance with the previous article in regards to the primary follicles (a single layer of cuboidal granulosa cells), sec- 
ondary follicles (surrounding of more than one layer of cuboidal granulosa cells without visible antrum), antral follicles (multiple layer of granulosa cells and 1-2 small spaces of antrum) and pre-ovulatory follicles (the largest follicle with cumulus granulosa cell layer surrounding the oocyte) (Kim, 2019).

\section{Immunofluorescence}

To identify the association between CYP1A2 expression and the follicle atresia, immunofluorescence with CYP1A2 and Fas, a common apoptosis inducer, was conducted. Preparation of slides until antigen retrieval step was conducted in a same manner with IHC. Then the slides were washed with PBST, blocked with $2 \%$ normal goat serum (Vector Laboratories) for $1 \mathrm{~h}$ at RT and incubated with a mouse polyclonal anti-CYP1A2 antibody (1:50 dilution with $2 \%$ normal goat serum) supplemented with a rabbit polyclonal anti-Fas antibody (1:1,000 dilution with $2 \%$ normal goat serum) at $4{ }^{\circ} \mathrm{C}$ for overnight. The slides were washed with PBS, incubated with a goat anti-mouse IgG conjugated with Alexa Fluor 594 (1:1,000 dilution with PBS) supplemented with a goat anti-rabbit conjugated with Alexa Fluor 488 (1:1,000 dilution with PBS) at RT for 60 min, counterstained with 4',6-diamidino-2-phenylindole (DAPI) for $5 \mathrm{~min}$ and mounted with VectaShield (Vector Laboratories). The slides were observed in fluorescent microscope (Moticam Pro 20A) at the proper wavelengths.

\section{Statistical analysis}

Student's T-test or Kruskal-Wallis test with Bonferroni correction was applied using PASW Statistics 18 (SPSS Inc., Chicago, IL, USA). A $p$ value of $<0.01$ was considered to be statistically significant.

Table 1. Confirmation of prepuberty and puberty

\begin{tabular}{lcc}
\hline & Prepuberty $(\mathrm{n}=\mathbf{8})$ & Puberty $(\mathrm{n}=\mathbf{8})$ \\
\hline Postnatal day (PND) & 24 & 51 \\
Body weight (BW) & $60.3 \pm 1.2 \mathrm{~g}$ & $177.0 \pm 6.5 \mathrm{~g}^{*}$ \\
Vaginal opening (VO) & $0 / 8$ & $8 / 8$ \\
Corpus luteum (CL) & $0 / 3$ & $3 / 3$ \\
\hline
\end{tabular}

The values are described as Mean \pm SD $(B W)$ or positive number/total number of animals (VO and $\mathrm{CL}$ ). ${ }^{*}$ Superscript indicates significant difference between groups $(p<0.01)$. "Histological assessment is conducted in three animals from each group $(n=3)$.

\section{RESULTS}

\section{Confirmation of prepuberty and puberty}

In accordance with previous article, SD rats in PND 24 or 51 of the present study were expected as prepubertal or pubertal periods, respectively (Rivest, 1991). In addition, BW and VO were assessed prior to sacrificing animals (Table 1). Prepubertal group $(60.3 \pm 1.2 \mathrm{~g})$ exhibited significantly $(p<0.01)$ lower BW than pubertal group $(177.0 \pm 6.5 \mathrm{~g})$. All animals in prepubertal group $(0 / 8)$ did not present VO but all did in pubertal group (8/8), indirectly indicating the onset of puberty. In case of $\mathrm{H} \& \mathrm{E}$ staining, the corpus lutea, implying the ovulation after the onset of puberty, were not observable in prepubertal group (Fig. 1A) but revealed in pubertal group (red arrows in Fig. 1B). Therefore, it was confirmed that each group presented the puberty-related features in consistent with their ages.

\section{Western blotting for quantitative assessment of CYP1A2 expression}

The western blotting was conducted to assess quantitative expression of CYP1A2 in the ovaries; because CYP1A2 was mainly known as a hepatic enzyme, the liver tissue samples from prepubertal and pubertal groups were also employed in this assay as positive controls (Fig. 2A). Overall, the expression level of CYP1A2 in the ovaries was significantly $(p<0.01)$ weaker than that of the livers, regardless of the onset of puberty (Fig. 2B). However, in case of CYP1A2 expression in the ovary, there was a significant $(p<0.01)$ upregulation of CYP1A2 expression in pubertal group than prepubertal group (Fig. 2B).

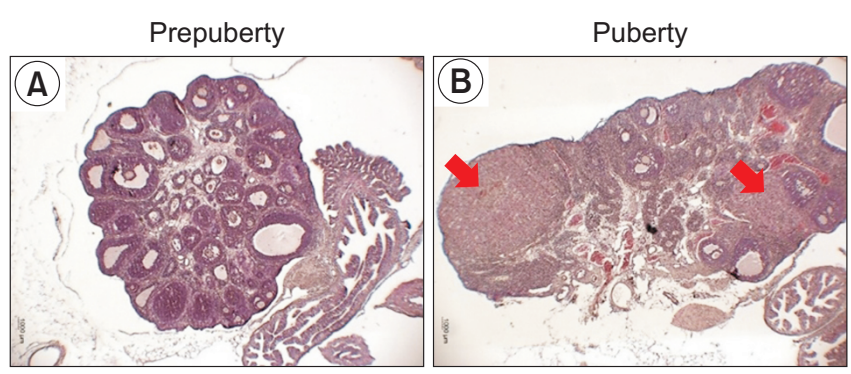

Fig. 1. Confirmation of prepuberty and puberty. Representative image of the prepubertal ovary $(A)$ and pubertal ovary with the corpus lutea (red arrows) (B). Magnification: $\times 40$; Bars: 1,000 $\mu \mathrm{m}$. 


\section{Immunohistochemistry of CYP1A2 in prepubertal and pubertal ovaries}

The expressions of CYP1A2 in both groups were analyzed by IHC (Fig. 3). Although the ovaries of both groups expressed CYP1A2 at the cells surrounding follicles, the patterns were dependent on the stage of developing follicle and the onset of puberty. At both groups, the primordial, primary and secondary follicles exhibited the negative expression of CYP1A2 (red arrows in Fig. 3A and 3D).
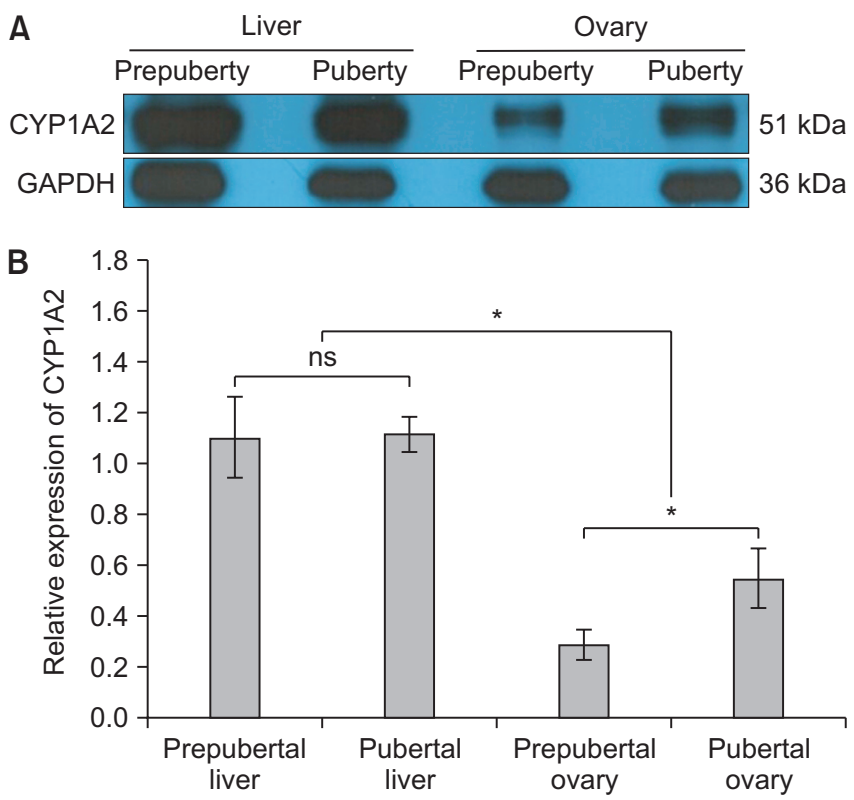

Fig. 2. Western blotting for assessment of CYP1A2 expression in prepubertal and pubertal group. Representative image of CYP1A2 expression in the ovary and liver (A). Graph for the expression level of CYP1A2 was presented as mean \pm SEM (B). ${ }^{*} p<0.01$ or ns indicated a significant difference or non-significance, respectively.
The CYP1A2-positive cells were observed from antral to pre-ovulatory follicles (blue arrows in Fig. 3B, 3C, 3E and $3 \mathrm{~F})$, and they were localized at the granulosa cell layer. When the CYP1A2-positive follicles were counted (\%), significantly $(p<0.01)$ higher number of follicles expressed CYP1A2 in pubertal group $(73.1 \pm 3.1 \%)$ than prepubertal group (41.0 \pm 10.5$)$ (Table 2). Collectively, these results suggested that CYP1A2 was strongly expressed at the granulosa cells in developing follicles (antral and preovulatory follicles) and affected by the onset of puberty.

\section{Immunofluorescence of CYP1A2 and Fas in the ovary}

To clarify the relationship between the CYP1A2 expression and atretic follicle, immunofluorescence assay of CYP1A2 and Fas was conducted in the ovary tissues (Fig. 4). As shown in Fig. 4A-4D, whereas the Fas-positive follicle, indicating the atretic follicle, co-expressed CYP1A2 (follicle on the left), the Fas-negative follicle did not express CYP1A2 (follicle on the right). In addition, the coexpression of Fas and CYP1A2 was evident in the granulosa and theca cell layers of pre-ovulatory follicle stage (Fig. 4E-4H). Based on these findings, it was concluded that CYP1A2 specifically expressed in the atretic follicle.

Table 2. Counting for CYP1A2-positive follicles in prepubertal and pubertal ovaries

\begin{tabular}{lcc}
\hline & Prepuberty $(n=3)$ & Puberty $(n=3)$ \\
\hline CYP1A2-positive follicle (\%) & $41.0 \pm 10.5$ & $73.1 \pm 3.1^{*}$ \\
\hline
\end{tabular}

The values are described as Mean \pm SD. *Superscript indicates significant difference between groups $(p<0.01)$.
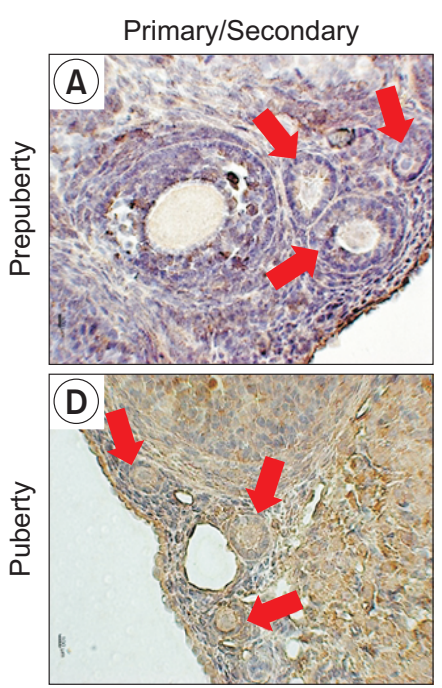
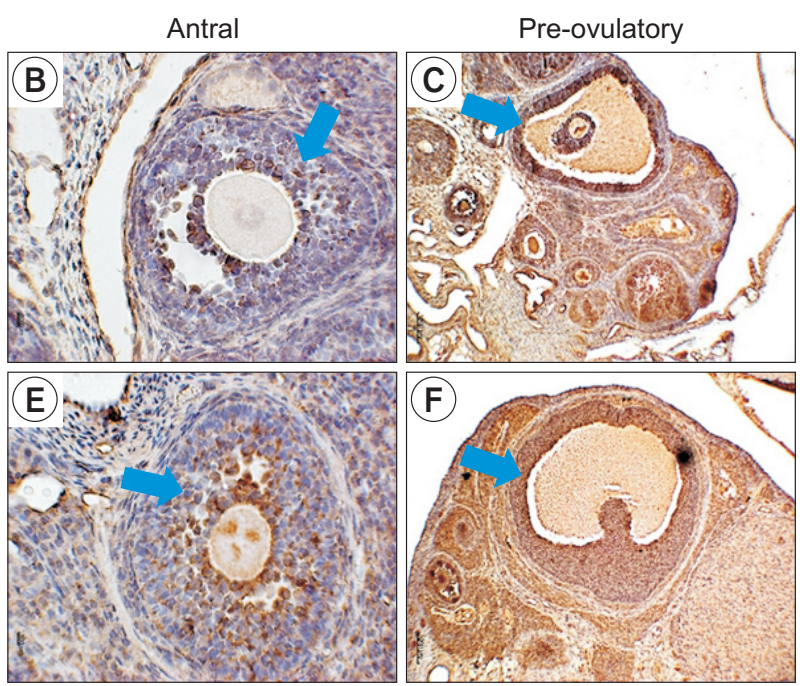

Fig. 3. Immunohistological expression of CYP1A2 in prepubertal and pubertal ovaries. The negative expressions of CYP1A2 at the primordial, primary and secondary follicles in both groups (red arrows in A and D). The CYP1A2positive cells, localized at the granulosa cells, from antral follicles (blue arrows in Fig. B and E) to pre-ovulatory follicles (blue arrows in $\mathrm{C}$ and $\mathrm{F}$ ). Magnification: $\times 400(A, B, D$ and $E)$ or $\times 100(C$ and $F)$; Bars: $100 \mu \mathrm{m}(\mathrm{A}, \mathrm{B}, \mathrm{D}$ and $\mathrm{E})$ or $1,000 \mu \mathrm{m}$ $(\mathrm{C}$ and $\mathrm{F})$. 

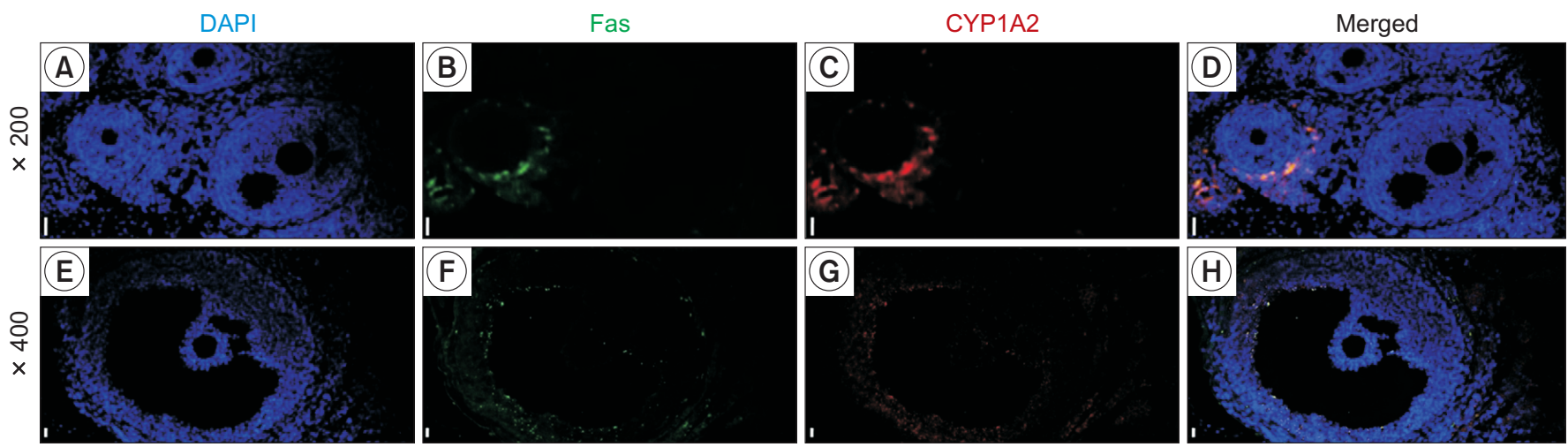

Fig. 4. Immunofluorescence of CYP1A2 and Fas in the ovary. The nucleus, Fas-positive cells and CYP1A2-positive cells were immunologically stained with DAPI ( $\mathrm{A}$ and $\mathrm{E}$, blue), Alexa Fluor 488 (B and F, green) and Alexa Fluor 594 ( $\mathrm{C}$ and $\mathrm{G}$, red), respectively. Magnification: $\times$ $200(A-D)$ or $\times 400(E-H)$; Bars: $100 \mu \mathrm{m}$.

\section{DISCUSSION}

It has been addressed that CYP1A2 is mainly expressed at the liver and plays a role in biotransformation of exogenous substances and metabolism of endogenous hormones (Mikhailova et al., 2006; Sugiyama et al., 2019). However, recent articles suggest that CYP1A2 is also expressed in other tissues such as the pancreas and lung (Vukovic et al., 2016). Therefore, we studied the localization of CYP1A2 expression depending on the onset of puberty of rats. Here, we demonstrated that CYP1A2 expression was mainly localized at the atretic follicular cells and affected by the onset of puberty.

The expression of CYP1A2 in the ovary has shown controversial and opposed results. Especially, some articles reported absence of CYP1A2 in the ovary. Via in situ hybridization, CYP1A2 mRNA was measurable in the liver at intact mouse and 3-methylcholanthrene (3MC; an Ah receptor ligand) could induce CYP1A2 mRNA expression in the liver, lung and duodenum; even though the ovaries were included as specimen in this study design, the expression of CYP1A2 in the ovary was negative (Dey et al., 1999). In addition, when the ovarian expressions of CYP isoforms were investigated in rats under diestrus or proestrus cycle, CYP1A2, CYP1A1, CYP2B1, CYP2C12, CYP2E1, CYP3A1, CYP3A2 and CYP4A1 were not detectable in the ovarian microsomes regardless of estrus cycles (Lee et al., 2012). In contrast, several articles have reported opposed results, detection of CYP1A2 expression in the ovary tissue. Positive expressions of CYP1A2 in ovaries and testes of domestic cats were identified using polymerase chain reaction (PCR) (Sugiyama et al., 2019). Moreover, the ovarian surface epithelium (OSE) of mouse was positive for CYP1A2, CYP1A1, CYP1B1 and CYP2C29 expression via PCR assay (Symonds et al., 2006). In the present study, we aimed to clarify localization of CYP1A2 expression in prepubertal and pubertal ovaries in rats. In agreement with the latter two articles, expressions of CYP1A2 in the ovary of rat were identified (Fig. 2 and 3, and Table 2). Especially, the intensity and localization of CYP1A2 expression were dependent on the onset of puberty and the stage of folliculogenesis; the expressions were negative at primordial, primary and secondary follicles at both prepubertal and pubertal groups, but strongly positive in the granulosa cells of antral and pre-ovulatory follicles. The reasons for the different findings in the present study compared to former two studies are still unknown, but such variations may be caused by differences of inducing substance, species, strain, age, gender and assay method (Dey et al., 1999; Symonds et al., 2006). For instance, human-mouse difference in the tissue specificity of CYP1A2 expression was observed when 3MC induced CYP1A2 expression in mouse lung but did not in human lung (Omiecinski et al., 1990; Dey et al., 1999). In addition, while CYP1A2 was detectable in the brain and colon of human, it was negative at the same set of tissues in mouse (Mercurio et al., 1996; Dey et al., 1999).

Numerous articles have contributed to reveal the classification of CYP family (e.g., CYP1, CYP2), its subfamily (e.g., CYP1A, CYP1B) and their isoform (e.g., CYP1A1, CYP1A2), and it is available to approach more than 18,000 sequences of CYPs today (Guengerich, 2013; McDonnell and Dang, 2013). Therefore, now it is important to reveal the function of each CYP in the body to under- 
stand maintenance of homeostasis, pharmacogenetics, carcinogenesis, molecular epidemiology and bioremediation. Especially, the study for function of CYPs has mostly been focused on the liver as the primary site of enzymatical metabolism of endogenous and exogenous substances (McDonnell and Dang, 2013). However, several CYP isoforms were detectable in not only the liver but also other organs such as the lung, prostate, adrenal gland, placenta and kidney (Nishimura et al., 2003). In case of CYP1A2, its expression was identified in the pancreas and lung as well as the ovary during the present study (Fig. 2 and 3) (Vukovic et al., 2016). Aside from CYP1A2, there have been several reports described expression and alteration of various CYP subfamilies or isoforms in the ovary. It was demonstrated that mouse OSE expressed CYP1A1, CYP1B1, CYP1A2 and CYP2C29, which are capable of metabolizing estrogen; in particular, the expression of CYP1B1 was elevated under estrogen treatment but CYP1A1 did not (Symonds et al., 2006). Whereas CYP1A1, CYP1A2, CYP2B1, CYP2C12, CYP2E1, CYP3A1, CYP3A2 and CYP4A1 were not detectable, CYP1B1 was expressed in the ovarian microsomes from rat (Lee et al., 2012). In addition, the ovarian CYP1B1 of rat was increasingly expressed during evening of proestrus than other days, possibly due to metabolism of serum estradiol (Dasmahapatra et al., 2002). In human, several types of CYP subfamilies and isoforms (CYP1A1, CYP2A, CYP2B, CYP2F1, CYP2R1, CYP2U1, CYP3A5, CYP3A7, CYP3A43, CYP4Z1, CYP26A1 and CYP51) were immunoreactively positive in the healthy ovary (Downie et al., 2005). When ovarian fractions derived follicles were assessed by PCR, all follicles expressed CYP2E1, CYP2A and CYP2B even if there were follicle size-dependent differences (Cannady et al., 2003). In case of granulosa cell of pig, the expression of CYP1A1 was extensively induced after treatment of $3 \mathrm{MC}$ or dexamethasone (Leighton et al., 1995). Likewise, it is true that the expression of CYP is abundant across various tissues including the ovary and the expression pattern is dependent on the role of organs and several factors.

During the ovarian folliculogenesis, follicular cells in the primordial, primary, secondary and antral follicles morphologically are observed as one layer of flat granulosa cells, one layer of cuboidal granulosa cells, more than one layer of cuboidal granulosa cell without antrum and multiple layers of proliferating granulosa cells with presence of theca cells and small antrum, respectively. And fully formed antrum with the mural granulosa cells and theca cells is identified in the pre-ovulatory follicles (Kim, 2019; Gershon and Dekel, 2020). During this folliculogenesis, it has been well clarified that only the limited numbers of developing follicles can ovulate but the rest undergo atresia at the various developing stages of ovarian follicle, initiated from apoptosis of the granulosa cell (Yu et al., 2004; Li et al., 2016; Zhou et al., 2019). While the granulosa cell layer is aligned along the follicular basal lamina in the developing healthy follicles, apoptotic granulosa cells is appeared from even early stage of follicle atresia; the apoptosis of granulosa cells is gradually extended to the whole follicle and reached to the theca cell layer, and finally the majority of granulosa cell layer was severely disrupted with elimination of follicle (Matsuda et al., 2012). The profile for expression of proteins extracted from the granulosa cells of healthy and atretic follicles in the porcine ovary presented that 399 from 4,591 proteins were differentially expressed; granulosa cells from atretic follicles exhibited upregulation of proteolysis, protein destabilization, phagocytosis and engulfment (Shan et al., 2020). In another study using the porcine ovary, 450 differentially expressed genes were identified between healthy and atretic follicles (Zhang et al., 2018). Moreover, atretic granulosa cells increasingly expressed Fas, FasL, TNF- $\alpha$, TNFR and pro-apoptotic protein (BAK), and decreased insulin-like growth factor (IGF) (Yu et al., 2004; Matsuda et al., 2012; Zhang et al., 2018). Furthermore, granulosa cells in the atretic follicles in goats exhibited higher number of apoptotic body $(51 \pm 2 \%)$ than those from healthy developing follicles $(13 \pm 2 \%)$ and slightly atretic follicles $(32 \pm 2 \%)$ by terminal deoxynucleotidyl transferase-mediated dUTP nick end labeling (TUNEL), and the concentration of estrogen in the follicular fluid was lower in atretic follicles than others (Yu et al., 2004). In case of rodents, granulosa cells and theca cells of atretic follicles positively presented Fas during prepubertal period from PND 17 to 35, and 192 upregulated and 116 downregulated gene expressions were observed between the transcriptomes from cyclophosphamideinduced atretic follicles and controls (Takagi et al., 2007; Lin et al., 2018). Collectively, it has been proven that the granulosa cells are undergone for several alterations during follicular atresia. In the present study, the expression of CYP1A2 was strongly localized in proliferated granulosa cell layers in not early follicles (primordial, primary 
and secondary follicles) but the developing follicles (antral and pre-ovulatory follicles) and dependent on the puberty (Fig. 2 and 3, and table 2). In addition, we found that Fas and CYP1A2 were co-expressed in an atretic follicle (Fig. 4 ), indicating change of the ovarian steroidogenesis pathway in the atretic follicles. Further study is still necessary but now we hypothesize that the atretic follicles may express CYP1A2 to metabolize residue of hormones because atretic follicles do not require the reproductive hormones for follicle development.

In conclusion, the present study hypothesized that CYP1A2 expressed in the ovary because ovarian cycle is tightly and directly related with reproductive hormones. Collectively, we demonstrated that CYP1A2 expression was localized at the granulosa cells in the atretic follicles, and affected by the onset of puberty. These findings may have important implications for the fields of reproductive biology.

\section{CONFLICTS OF INTEREST}

No potential conflict of interest relevant to this article was reported.

\section{ACKNOWLEDGEMENTS}

This work was supported by a grant from the National Research Foundation (NRF) of Korea, funded by the government of the Republic of Korea (NRF-2020R1F1A1076723).

\section{AUTHOR CONTRIBUTIONS}

Conceptualization: W.J.L., S.J.K., Y.S.K.

Funding Acquisition: W.J.L.

Animal works: J.C.H., B.J.P, H.D.K.

Molecular works: J.C.H., S.M.B., S.W.L., J.K.P., S.G.B., R.H.J, M.J.

Writing original draft: J.C.H.

Review \& editing: W.J.L., S.J.K., S.H.Y.

\section{AUTHOR'S POSITION AND ORCID NO.}

JC Hwang, MS Candidate, https://orcid.org/0000-0002-1741-3405

BJ Park, MS, https://orcid.org/0000-0003-1901-0869
HD Kim, PhD Candidate, https://orcid.org/0000-0003-0917-9863

SM Baek, PhD Candidate, https://orcid.org/0000-0002-7222-6186

SW Lee, PhD Candidate,

https://orcid.org/0000-0002-7678-9242

RH Jeon, PhD,

https://orcid.org/0000-0003-3174-1197

M Jang, Assistant Professor, https://orcid.org/0000-0002-2188-1906

SG Bae, Assistant Professor, https://orcid.org/0000-0001-9487-5665

SH Yun, Assistant Professor, https://orcid.org/0000-0002-9027-3859

JK Park, Associate Professor, https://orcid.org/0000-0003-4876-1055

YS Kwon, Professor,

https://orcid.org/0000-0002-6489-0327

SJ Kim, Professor, https://orcid.org/0000-0002-8521-8898

WJ Lee, Assistant Professor, https://orcid.org/0000-0003-1462-7798

\section{REFERENCES}

Cannady EA, Dyer CA, Christian PJ, Sipes IG, Hoyer PB. 2003. Expression and activity of cytochromes P450 2E1, 2A, and 2B in the mouse ovary: the effect of 4-vinylcyclohexene and its diepoxide metabolite. Toxicol. Sci. 73:423-430.

Dasmahapatra AK, Trewin AL, Hutz RJ. 2002. Estrous cycle-regulated expression of CYP1B1 mRNA in the rat ovary. Comp. Biochem. Physiol. B Biochem. Mol. Biol. 133:127-134.

Dey A, Jones JE, Nebert DW. 1999. Tissue- and cell type-specific expression of cytochrome P450 1A1 and cytochrome P450 $1 \mathrm{~A} 2 \mathrm{mRNA}$ in the mouse localized in situ hybridization. Biochem. Pharmacol. 58:525-537.

Downie D, McFadyen MC, Rooney PH, Cruickshank ME, Parkin DE, Miller ID, Telfer C, Melvin WT, Murray GI. 2005. Profiling cytochrome $\mathrm{P} 450$ expression in ovarian cancer: identification of prognostic markers. Clin. Cancer Res. 11:73697375.

Gaytan F, Morales C, Leon S, Heras V, Barroso A, Avendaño MS, Vazquez MJ, Castellano JM, Roa J, Tena-Sempere M. 2017. Development and validation of a method for precise dating of female puberty in laboratory rodents: the puberty ovarian maturation score (Pub-Score). Sci. Rep. 7:46381.

Gershon E and Dekel N. 2020. Newly identified regulators of ovarian folliculogenesis and ovulation. Int. J. Mol. Sci. 21:4565.

Guengerich FP. 2013. New trends in cytochrome p450 research 
at the half-century mark. J. Biol. Chem. 288:17063-17064.

Kim SJ. 2019. The chronic and unpredictable stress suppressed kisspeptin expression during ovarian cycle in mice. J. Anim. Reprod. Biotechnol. 34:40-49.

Lee HJ, Jeon RH, Park BJ, Jang SJ, Lee SL, Rho GJ, Kim SJ, Lee WJ. 2019. Differentiation inductions altered telomere length and telomerase activity in human dental pulp-derived mesenchymal stem cell. J. Anim. Reprod. Biotechnol. 34:93-99.

Lee SY, Oh SJ, Yun KU, Kim HM, Kim BH, Lee K, Kim SK. 2012. Expression of hepatic and ovarian cytochrome P450 during estrous cycle in rats. Arch. Toxicol. 86:75-85.

Leighton JK, Canning S, Guthrie HD, Hammond JM. 1995. Expression of cytochrome P450 1A1, an estrogen hydroxylase, in ovarian granulosa cells is developmentally regulated. J. Steroid Biochem. Mol. Biol. 52:351-356.

Li J, Gao H, Tian Z, Wu Y, Wang Y, Fang Y, Lin L, Han Y, Wu S, Haq I, Zeng S. 2016. Effects of chronic heat stress on granulosa cell apoptosis and follicular atresia in mouse ovary. J. Anim. Sci. Biotechnol. 7:57.

Lin J, Zheng J, Zhang H, Chen J, Yu Z, Chen C, Xiong Y, Liu T. 2018. Cytochrome $\mathrm{P} 450$ family proteins as potential biomarkers for ovarian granulosa cell damage in mice with premature ovarian failure. Int. J. Clin. Exp. Pathol. 11:42364246.

Lu J, Shang X, Zhong W, Xu Y, Shi R, Wang X. 2020. New insights of CYP1A in endogenous metabolism: a focus on single nucleotide polymorphisms and diseases. Acta Pharm. Sin. B 10:91-104.

Matsuda F, Inoue N, Manabe N, Ohkura S. 2012. Follicular growth and atresia in mammalian ovaries: regulation by survival and death of granulosa cells. J. Reprod. Dev. 58:4450.

Mayer C, Acosta-Martinez M, Dubois SL, Wolfe A, Radovick S, Boehm U, Levine JE. 2010. Timing and completion of puberty in female mice depend on estrogen receptor alphasignaling in kisspeptin neurons. Proc. Natl. Acad. Sci. U. S. A. 107:22693-22698.

McDonnell AM and Dang CH. 2013. Basic review of the cytochrome p450 system. J. Adv. Pract. Oncol. 4:263-268.

Mercurio MG, Shiff SJ, Galbraith RA, Sassa S. 1995. Expression of cytochrome P450 mRNAs in the colon and the rectum in normal human subjects. Biochem. Biophys. Res. Commun. 210:350-355.

Mikhailova ON, Gulyaeva LF, Prudnikov AV, Gerasimov AV, Krasilnikov SE. 2006. Estrogen-metabolizing gene polymorphisms in the assessment of female hormone-dependent cancer risk. Pharmacogenomics J. 6:189-193.

Nishimura M, Yaguti H, Yoshitsugu H, Naito S, Satoh T. 2003. Tissue distribution of mRNA expression of human cytochrome $\mathrm{P} 450$ isoforms assessed by high-sensitivity real-time reverse transcription PCR. Yakugaku Zasshi 123:369-375.

Omiecinski CJ, Redlich CA, Costa P. 1990. Induction and developmental expression of cytochrome P450IAl messenger RNA in rat and human tissues: detection by the polymerase chain reaction. Cancer Res. 50:4315-4321.

Rivest RW. 1991. Sexual maturation in female rats: hereditary, developmental and environmental aspects. Experientia 47:1027-1038.

Shan X, Yu T, Yan X, Wu J, Fan Y, Guan X, Fang F, Lin Y, Zhang Y, Li Y, Liu Y. 2020. Proteomic analysis of healthy and atretic porcine follicular granulosa cells. J. Proteomics 31:104027.

Sugiyama S, Uno Y, Amano T, Kitazawa T, Teraoka H. 2019. Genetic diversity of cytochrome P450 1A2 with different metabolic activities in domestic cats. J. Vet. Med. Sci. 81:980-982.

Symonds DA, Miller KP, Tomic D, Flaws JA. 2006. Effect of methoxychlor and estradiol on cytochrome p450 enzymes in the mouse ovarian surface epithelium. Toxicol. Sci. 89:510514.

Takagi K, Yamada T, Miki Y, Umegaki T, Nishimura M, Sasaki J. 2007. Histological observation of the development of follicles and follicular atresia in immature rat ovaries. Acta Med. Okayama 61:283-298.

Uenoyama Y, Inoue N, Maeda KI, Tsukamura H. 2018. The roles of kisspeptin in the mechanism underlying reproductive functions in mammals. J. Reprod. Dev. 64:469-476.

Vukovic V, Ianuale C, Leoncini E, Pastorino R, Gualano MR, Amore R, Boccia S. 2016. Lack of association between polymorphisms in the CYP1A2 gene and risk of cancer: evidence from meta-analyses. BMC Cancer 16:83.

Wijnen PA, Op den Buijsch RA, Drent M, Kuijpers PM, Neef C, Bast A, Bekers O, Koek GH. 2007. Review article: the prevalence and clinical relevance of cytochrome $\mathrm{P} 450$ polymorphisms. Aliment. Pharmacol. Ther. 26 Suppl 2:211-219.

Yu YS, Sui HS, Han ZB, Li W, Luo MJ, Tan JH. 2004. Apoptosis in granulosa cells during follicular atresia: relationship with steroids and insulin-like growth factors. Cell Res. 14:341346.

Zhang J, Liu Y, Yao W, Li Q, Liu H, Pan Z. 2018. Initiation of follicular atresia: gene networks during early atresia in pig ovaries. Reproduction 156:23-33.

Zhou J, Peng X, Mei S. 2019. Autophagy in ovarian follicular development and atresia. Int. J. Biol. Sci. 15:726-737. 